\title{
Evaluation of Current Antioxidant Profile in Semen
}

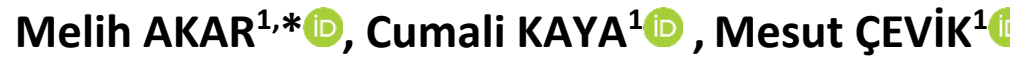

${ }^{1}$ Ondokuz Mayıs University, Faculty of Veterinary Medicine, Department of Reproduction and Artificial Insemination, Samsun, Turkey.

*Corresponding author

\section{Article History}

Received: 5 September 2020

Accepted: 23 March 2021

First Online: 15 April 2021

\section{Corresponding Author}

Tel: 0 (362) 312-1919/ 1355

E-mail: maveteriner55@gmail.com

\begin{abstract}
The freezing and storage of the sperm are used cryopreservation of germplasm in livestock breeding, genetic improvement of indigenous species, preservation of rare races, successful tolerance to environmental changes and international germplasm exchanges. Both the freezing and thawing process causes large changes in the volume of the cell fluid. Spermatozoon removes most of its cytoplasm at differentiation stages and lacks the cytoplasmic component that contains antioxidants that counteract the harmful effect of reactive oxygen species and lipid peroxidation. Therefore, the sensitivity of spermatozoa to lipid peroxidation increases during the freezing and thawing of the sperm, which creates a significant mechanical stress on the cell membrane. Oxidative stress is caused by oxygen and oxygen-derived oxidants, commonly known as ROS, and is known as an imbalance between the ability of biological systems to easily detoxify or repair damaged reagents. Uncontrolled ROS production, which exceeds the antioxidant capacity of seminal plasma, causes oxidative stress that is harmful to spermatozoa. All cellular components, including lipids, proteins, nucleic acids, and sugars, are potential targets of oxidative stress. Antioxidants control the chemical degradation of the substrate caused by oxidation, neutralizing free radicals, thereby it is used to minimize the risk of damage to spermatozoa during cryopreservation.
\end{abstract}

\section{Introduction}

Mammalian spermatozoa have a high energy demand to function. Spermatozoa contain approximately 50 to 75 mitochondria. The production of free radicals called hydroxyl radicals $(\bullet \mathrm{OH})$, superoxide anion $\left(\bullet \mathrm{O}^{2-}\right)$, hydrogen peroxide $\left(\mathrm{H}_{2} \mathrm{O}_{2}\right)$, and nitric oxide (NO) containing reactive oxygen species occurs in spermatozoon, like any other cell that performs aerobic metabolism (Bansal and Bilaspuri, 2010a). These ROSs are highly reactive molecules since their outer shell has an unpaired electron. In addition, they have a very short half-life between nanoseconds and milliseconds. ROS is formed by natural cell activity and participates in the normal cell cycle. Gametes are often susceptible to attack by reactive oxygen species (ROS), and manipulating gametes in vitro during assisted reproductive techniques can cause ROS to be generated by cells and exposed to ROS at supraphysiological levels (Agarwal et al., 2014). However, oxidative stress (OS) is called when the ROS production exceeds the physiological range and oxidants become more than antioxidants. The resulting OS production causes harmful effects that result in the oxidation of lipids, proteins, carbohydrates, and nucleotides (Birben et al., 2012). ROS formation in spermatozoa is likewise a natural physiological process and affects essential reproductive processes such as gametes, spermatozoon-oocyte interactions, implantation, and early development of embryos.

A common and significant technique, sperm cryopreservation provides a valuable therapeutic alternative in the field of assisted reproduction (Hezavehei et al., 2018). Osmotic stress that occurs during 
cryopreservation results from changes in cell volume due to water movement and dissolution along the spermatozoon plasma membrane and this causes ROS formation (Ball, 2008). Numerous mitochondria, low cytoplasm, and low antioxidant content in the specific cell structure of spermatozoon and plasma membrane make spermatozoon vulnerable to damage caused by free radicals (Bollwein et al., 2008). Antioxidants act as the main defense factors against oxidative stress caused by free radicals (Silva et al., 2011). For this reason, an antioxidant is added effectively to sperm freezing diluents.

\section{Oxidative Stress}

Oxidative stress (OS) is caused by oxygen and oxidants, generally referred to as the reactive oxygen species (ROS). It is called the imbalance between the ability of biological systems to detoxify reactive intermediates easily or to repair the resulting damage easily. Uncontrolled production of ROS, which exceeds seminal plasma's antioxidant capacity, results in OS, which is harmful to sperm cells. The potential targets of oxidative stress include all cellular components including lipids, proteins, nucleic acids, and sugars (Bansal and Bilaspuri, 2010a).

The main reason for the occurrence of oxidative stress in sperms is the depletion of seminal antioxidants and the production by spermatozoon of excess free radicals (Wathes et al., 2007). In immature spermatozoa can produce a significant amount of ROS that negatively correlates with semen quality (Agarwal and Majzoub,
2017). Nevertheless, it is not yet clear what mechanisms increase oxidative stress in frozen-thawed sperm. Some authors attribute this to the depletion of antioxidative enzymes (Stradaioli et al., 2007), while others suggest that osmotic stress induces oxidative stress during sperm freezing and thawing (McCarthy et al., 2010). Hyperosmotic cell swelling can then induce NADPH (Nicotinamide Adenine Dinucleotide Phosphate) oxidase in somatic cells, activating membrane-bound phospholipase A2, which allows free polyunsaturated fatty acids to form as arachnoid acid, resulting in an increase in production of $\mathrm{O}_{2}$ (Lambert et al., 2006).

\section{Effects of Oxidative Stress on Spermatozoa}

The adverse effects of oxidative stress on spermatozoon activity have been seen in several ways as it affects many essential molecules, including lipids, proteins, and DNA due to excessive ROS (Bollwein and Bittner, 2018). Through the presence of small molecules and the action of antioxidant enzymes in their cytoplasm, most cells may prevent oxidative stress, but through transcriptional activation of genes corresponding to these proteins, spermatozoa are exceptions. The silent nature of this cell also demonstrates that it cannot reverse the changes that affect it and in particular the damage to its genetic material. In other words, if spermatozoa are unable to protect themselves effectively against oxidative stress, death due to necrosis or apoptosis is the only option for this cell if subjected to acute stress.

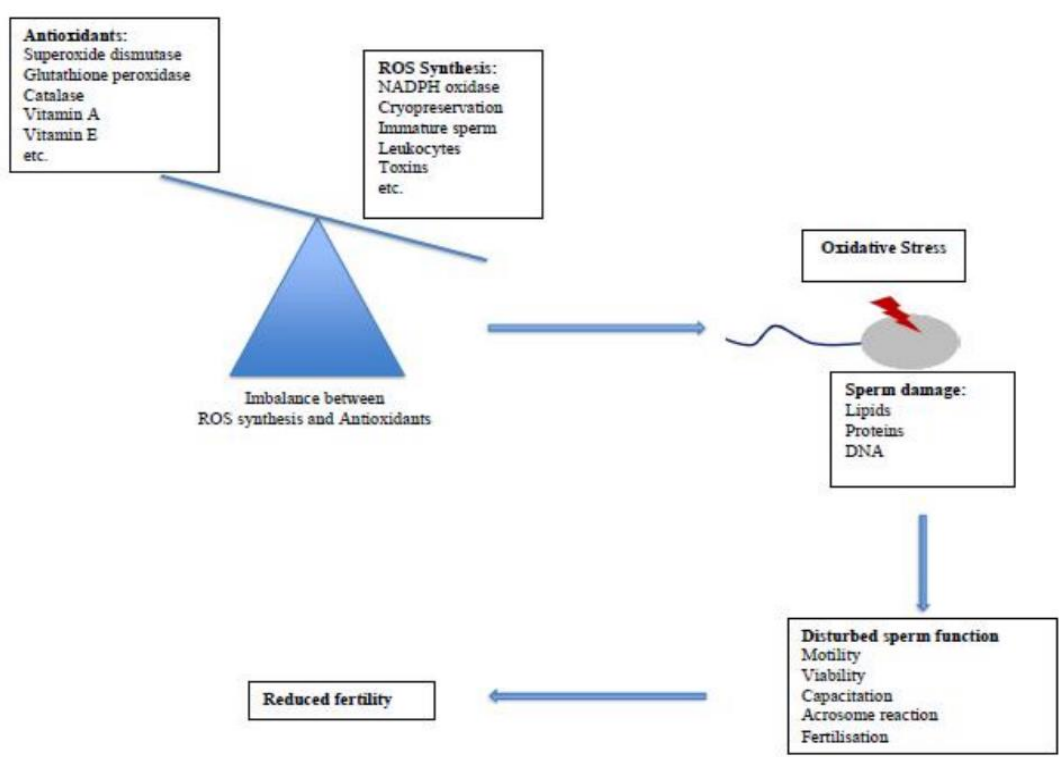

Figure 1. Oxidation sources in semen and their role in disturbances of sperm quality and male fertility (Bollwein and Bittner, 2018). 
This state of fragility worsens with the unique lipid composition of the spermatozoon plasma membrane in relation to oxidative stress (Drevet and Aitken, 2020).

\section{Lipid Peroxidation}

The damage mechanism caused by ROS in spermatozoa involves an oxidative attack that leads to the initiation of lipid peroxidation (LPO) on spermatozoon membrane lipids (Aitken, 2017). Lipid peroxidation produces a variety of lipid metabolites, including lipid peroxyl radicals, alkoxyl radicals, malondialdehyde, 4-hydroxynonenal, and acrolein. Lipid peroxides are self-produced in the plasma membrane of spermatozoa and released under the action of phospholipase $\mathrm{A} 2$, and are also reduced by glutathione peroxidase to phospholipids. The membranes of mammalian spermatozoa are abundant in polyunsaturated fatty acids (PUFAs) and are responsive to lipid peroxidation-mediated oxygen damages. The presence of PUFAs gives the flexibility of the membranes to help the membrane enter into the fluidity-related membrane fusion events and the capacity of sperm to fertilize. Unfortunately, the presence of double bonds in these molecules makes them vulnerable to free radical attacks and LPO initiation. This leads to the impaired membrane and morphological integrity, impaired cell function, impaired sperm mobility, impaired fertility of spermatozoa with oocytes, and induction of apoptosis of spermatozoa (Bucak et al., 2010). In addition, lipid peroxidation is known to reduce the mobility of spermatozoa. Mechanisms include modulating the function of ion channels with changes in membrane structure (Bollwein and Bittner, 2018) and the development of lipid metabolites with flagellar axonemal proteins and proteins mediated by mitochondrial electrons. Modulation of mitochondrial proteins interferes with the transport of mitochondrial electrons and causes electrons to flow out. And these, combine with oxygen in a vicious loop to create additional ROS (Moazamian et al., 2015).

\section{Free Radicals}

Free radicals are short-lived chemical reactive intermediates with one or more unpaired electrons. These unpaired electrons move into nearby cellular structures so cause damage to the cells. In this case, amino acids in proteins or nucleic acids induce oxidation of the cell membrane lipids (Ifeanyi, 2018). Free radicals are also required for normal cell proliferation, differentiation, and intracellular signaling that occurs in the process of migration. Excessive free radical formation often results in a spermiogenesis error, which causes abnormally high levels of cytoplasmic retention to be released from the germinal epithelium (Sanocka and Kurpisz, 2004).

\section{Reactive Oxygen Types (ROS)}

Reactive oxygen species (ROS) contain radicals of oxygen as well as hydrogen peroxide $\left(\mathrm{H}_{2} \mathrm{O}_{2}\right)$, superoxide anion $\left(\bullet \mathrm{O}_{2}-\right)$, and hypochlorous acid $(\mathrm{HOCl})$ and highly reactive forms of $\mathrm{O}_{2}$ which do not contain unpaired (non-radicals) electrons (Bollwein and Bittner, 2018). ROS also represents a wide category of molecules indicating that radicals (hydroxyl ion, superoxide, nitric oxide, peroxyl, etc.) and non-radicals (ozone, single oxygen, lipid peroxide, hydrogen peroxide) and oxygen derivatives are collected. These molecules are unstable and try to capture a stabilizing electron, increasing the imbalance and leading to oxidation of other molecules (Drevet and Aitken, 2020).

ROS produced by sperm plays a significant role in normal physiological processes such as capacitation of spermatozoa, acrosome reaction, preservation of fertility capacity, and stabilization of the mitochondrial capsule in cattle (Desai et al., 2010; Gonçalves et al., 2010). Physiological ROS concentrations in vivo play a role in ensuring membrane fluidity, maintaining spermatozoa fertilization capability, and acrosome reaction (Bucak et al., 2010). Therefore, maintaining an appropriate ROS level is essential for the functionality of appropriate spermatozoon functions. The advanced antioxidant system counteracts the homeostasis of ROS and controls it.

\section{Protein Modifications}

Reactive oxygen species are thought to oxidize the side chains of amino acid residues, directly altering the proteins by supplying peptide bond splitting and covalent protein-protein cross-link formation. Oxidation may affect the conformation or activity of proteins. The thiol group is an example of this situation. Cysteine, the amino acid, contains a group of thiol and this can affect many proteins. In semen, the tyrosine phosphatase enzyme which plays an important role in the capacitation spermatozoa has a thiol group and is therefore vulnerable to ROS oxidation. Also, long-term oxidative stress can lead to excessive oxidation of thiol protamine groups, thereby causing hyper-condensation of DNA has a negative effect on function (Bollwein and Bittner, 2018).

\section{DNA Damage}

Free radicals may have many ways of damage to DNA. Double bonds of DNA bases and abstract hydrogen from deoxyribose sugar are linked to hydroxyl radicals (Cadet et al., 2003). Hydrogen isolation from deoxyribose carbon induces splits in the fiber and the base emissions. The ROS attack on the bases causes a large number of base changes (Bollwein and Bittner, 2018). One of the most common changes is guanine 
oxidation. Depending on the intensity of oxidative stress, there may be changes in DNA fragmentation (single or double chain breaks), from simple oxidation of bases (the most sensitive bases, guanosine, and adenosine) to oxidative stress. Other oxidative DNA events can also be observed, including the creation of specific regions and crosslinking of DNA-proteins. Due to the lack of a fully functioning DNA repair mechanism in mature spermatozoa, oocyte DNA repair systems (mainly post-fertilization oocyte base excision repair pathway) are needed to correct these oxidative changes. Also, in circumstances of moderate to low oxidative stress that does not cause DNA degradation, baseline oxidation may occur and must be corrected in this case (i.e., each oxidized base must be substituted with an unoxidized base) (Drevet and Aitken, 2020).

\section{Current Antioxidant Substances}

Antioxidants can be anti-ROS antioxidants (metal chelators or binding proteins such as lactoferrin) or antioxidants that eliminate existing ROS (such as vitamins C and E) (Hussain et al., 2018). It is classified into two groups according to its chemical properties: enzymatic antioxidants and non-enzymatic antioxidants (Félix et al., 2021). Enzymatic antioxidants are known as natural antioxidants; glutathione, glutathione peroxidase, glutathione reductase, catalase, and superoxide dismutase (SOD). These all join the spermatozoa's normal antioxidant defense mechanism. Non-enzymatic antioxidants or synthetic antioxidants or nutritional supplements include vitamin $\mathrm{C}$, and minerals such as vitamin $E$, zinc and selenium, taurine, hypotaurine, butylated hydroxytoluene (BHT), and melatonin. Non-enzymatic antioxidants are obtained from fruits or vegetables (Bansal and Bilaspuri, 2010b). Antioxidants also serve as motility-enhancing agents, thereby increasing sperm motility and the ability to fertilize. Additionally, antioxidants are not only used to preserve the spermatozoon membrane integrity but also to prevent membrane damage by pressing on lipid peroxidation and ROS development of embryos and oocytes (Uysal et al., 2000).

\section{Enzymatic Antioxidants}

\section{Glutathione}

Glutathione (GSH) the key protein thiol compound in mammalian cells is present in a variety of cells and has the capacity to react directly with ROS. Additionally, glutathione has a defensive role to exogenous antioxidants such as vitamin $\mathrm{C}$ and $\mathrm{E}$ active forms. (Atmaca, 2004). It also helps in reducing toxic $\mathrm{H}_{2} \mathrm{O}_{2}$ and hydroperoxides, thus shielding mammalian cells from ROS. Sulphydryl groups of GSH protect cells against oxidants, electrophiles, and free radicals (Agarwal et al., 2008). This function of GSH has been shown in vitro studies in which the frequency of the tail shot is maintained, LPO is decreased, and properties of the spermatozoon membrane are improved. Glutathione acts as protective agents in many types of cells against the negative effects of ROS-induced damage. In spermatozoa, it prevents the process of lipid peroxidation from damage to ROS. GSH also has a protective effect on normal acrosome integrity and also stabilizes sperm plasmalemma and improves motility (Bello et al., 2020).

Glutathione peroxidase (GSHPx) reduces lipoperoxides to alkyl alcohols and to hydrogen peroxide $\mathrm{H}_{2} \mathrm{O}$ by using glutathione (Amidi et al., 2016). Glutathione peroxidase primarily acts as an antioxidant cleaner in the epididymis and testicles (Mora-Esteves and Shin, 2013) and helps preserve the viability and motility of spermatozoa by supplying protection in the lipid components of the spermatozoon membrane (Bello et al., 2020). GSH can be regenerated from the oxidized form of glutathione reductase (GSR), the function of which is inducible to oxidative stress. In pigs, the application of 1 and $5 \mathrm{mM}$ GSH to semen diluent had founded beneficial effects on the quality of semen (Gadea et al., 2005). In ram semen, the use of GSH concentrations has had a beneficial impact on acrosome integrity between 2 and $5 \mathrm{~mm}$ (Silva et al., 2011).

\section{Superoxide Dismutase and Catalase}

SOD catalyzes the conversion of superoxide to oxygen and $\mathrm{H}_{2} \mathrm{O}_{2}$, which protects sperm from superoxide anions and thereby prevents LPO and enhances motility (Agarwal et al., 2008). Although SOD transforms the spontaneous superoxide anion to form $\left(\mathrm{O}^{2-} \bullet\right) \mathrm{O}_{2}$ and $\mathrm{H}_{2} \mathrm{O}_{2}$, as seen in the equation below, it converts catalase $\mathrm{H}_{2} \mathrm{O}_{2}$ to $\mathrm{O}_{2}$ and $\mathrm{H}_{2} \mathrm{O}$, so both SOD and catalase help eliminate ROS, which has the potential to damage spermatozoa and it plays an important role in reducing LPO (Bello et al., 2020).

Before the cryopreservation, dilution with extenders decreases the concentration of these components in seminal plasma and leaves sperm susceptible to oxidative stress (Martinez-Paramo et al., 2012). Adding SOD or CAT to the porcine sperm freezing extender, however, not only increases the sperm mobility and viability but also decreases post-thaw ROS production, which leads to an increase in the in vitro fertilization capacity of the dissolved sperm. Such results 
indicate that adding CAT and SOD to the extender increases the survival and in vitro viability of liquid semen storage (Bello et al., 2020).

\section{Non-Enzymatic Antioxidants}

Vitamin E

Vitamin E ( $\alpha$-tocopherol) is a chain-breaking antioxidant present in the membrane of the spermatozoon. It works by neutralizing $\mathrm{H}_{2} \mathrm{O}_{2}$ and quenching free radicals (Bansal and Bilaspuri, 2010b). It thus prevents the chain reactions that create lipid peroxide and protects the plasma of sperm from ROS damage (Bello et al., 2020). This also increases the function of other oxidizing agents (Mora-Esteves and Shin, 2013), thereby helping to preserve sperm mobility as well as morphology (Agarwal et al., 2008). Vitamin $\mathrm{E}$ supplementation during cryopreservation has a beneficial effect on sperm motility, mitochondrial membrane potential, and membrane integrity, depending on the fraction of the ejaculate (Bello et al., 2020). It has been reported that adding vitamin $E(5 \mu \mathrm{g} /$ $\mathrm{MI})$ in combination with $1 \%$ Nano-Se also increases sperm quality after thawing and enhances cock sperm oxidative variables (Safa et al., 2016).

Vitamin C

Vitamin C (Ascorbic acid) is a water-soluble chain breaker antioxidant, capable of clearing radicals of oxygen with low toxicity and high ability. Ascorbic acid secreted from seminal vesicles is the main antioxidant found in fertile men's seminal plasma, providing up to $65 \%$ of the total chain-breaking antioxidant capacity (Amidi et al., 2016). Vitamin C plays a significant role in the battle against oxidative stress in seminal plasma by reacting in extracellular fluid with $\mathrm{OH}-, \mathrm{O}_{2}-$ and $\mathrm{H}_{2} \mathrm{O}_{2}$, maintaining sperm viability, stability, and preventing sperm agglutination (>65\%) (Agarwal et al., 2008). Vitamin $C$ can also serve as a pro-oxidant and make radicals, in the presence of transition metals, more reactive and highly destructive. The addition of $2,5 \mathrm{mM}$ and $0,02-0,6 \mathrm{mM}$ of vitamin $\mathrm{C}$ to cattle and human sperm has been shown to have a detrimental effect on sperm motility in frozen-thawed bull sperm and in samples of human normozoospermic and asthenozoospermic, however, the subsequent application of $5 \mathrm{mM}$ revealed, however, an important protective effect against lipid peroxidation in frozen bovine quality semen (Amidi et al., 2016).

\section{Butylated Hydroxy Toluene (BHT)}

Butylated hydroxytoluene (BHT), also known as dibutylhydroxytoluene, is a non-enzymatic, synthetic analog of vitamin E. BHT is an organic soluble molecule used to stop autooxidation of the double lipid layer and spermatozoon membrane. BHT has properties of antioxidants, antivirals, and antimicrobials. Because spermatozoa are high targets for reactive oxygen species (ROS), the addition of BHT is beneficial for its role in free radical scavenging. BHT reacts with ROS and converts to hydroperoxides. It helps to avoid lipid peroxidation in biological membranes and by being used as a complement in different semen extender it increases the consistency of spermatozoa. BHT also acts as an antiviral agent and thus reduces the risk of viral disease transmission to the female animal during artificial insemination (Bello et al., 2020).

Farshad et al. (2011) demonstrated that the optimum concentration of BHT needed to optimally preserve ram semen during cryopreservation is $2-3 \mathrm{mM}$ $\mathrm{BHT}$. While the optimal concentration of $\mathrm{BHT}$ required for sperm survival varies depending on the animal species, it may range from 0.05-2.0 mM. Higher butylated concentrations of hydroxytoluene adversely affect the properties of freezing thawed semen and the process remains unclear. BHT concentrations of 0.5 and $2.0 \mathrm{mM} / \mathrm{mL}$ in buffaloes are the preferred quantities for sperm preservation, depending on the diluent and freezing stage (Bello et al., 2020).

\section{Carnitine}

Carnitine is an antioxidant that is water-soluble and typically obtained from dietary sources. This can contribute to sperm motility as a source of fuel by aiding the use of free fatty acids and preventing lipid oxidation (Mora-Esteves and Shin, 2013). Therefore, Carnitine protects spermatozoa's viability and motility by shielding the spermatozoon DNA and membranes from oxidative damage. L-carnitine has been shown to be efficient in protecting chicken sperm from apoptosis, loss of mitochondrial function, and DNA binding (Hussain et al., 2018).

Albumin

Through interacting with peroxyl radicals, albumin avoids chain reactions that produce more free radicals and thus maintains sperm motility and viability by reducing the production of ROS. Albumin is known to improve sperm and plasma membrane integrity stability and to protect the acrosomes of ram spermatozoa from temperature shock during freeze-thaw. Albumin can also increase sperm survival and fertility capacities in the female reproductive system before fertilization, and it has been shown to improve antioxidant catalase activity after frozen and thawed bull semen (Hussain et al., 2018). 
Zinc

Zinc is one of the important trace elements that cause infertility in its deficiency, as well as controlling events such as testicular development, spermatogenesis, steroidogenesis through the secretion of gonadotropic hormones, genetic expression of steroid receptors, testosterone synthesis, and adjustment of serum cholesterol levels. In zinc-deficient animals, zinc supplementation contributes to increased fertility by increasing semen concentration, motility, and spermatozoon membrane integrity, as well as reducing spermatozoon DNA damage. Zinc also contributes to the spermatozoon chromatin stability and DNA damage repair. Zinc affects the fluidity of lipids and therefore the stability of biological membranes. It plays a function in the formation of free oxygen radicals and can play a regulatory role in capacitation and acrosome reaction processes, sperm condensation of nuclear chromatin, and acrosine activity (Dorostkar et al., 2014). Although the addition of higher zinc concentrations (0.576 and $1.152 \mathrm{mg} / \mathrm{L}$ ) to the extender is detrimental to spermatozoa, Dorostkar et al. (2014) reported that the addition of zinc sulphate $(0.288 \mathrm{mg} / \mathrm{L})$ resulted in improvement in the preservation of spermatozoon quality (progressive motility, viability, membrane integrity, and total antioxidant capacity) in freezing processes, and also caused less DNA damage affecting the cell membrane after semen freeze-thaw and this provides a higher fertility rate.

Zinc-nanoparticles are also among the metal nanoparticles used successfully to improve the quality of sperm and are considered an important element of spermatogenesis since it enhances the quality of sperms. In young rams, the addition of $50 \mathrm{mg} / \mathrm{kg}$ or 100 $\mathrm{mg} / \mathrm{kg}$ Zn-nanoparticle in the diet improved the efficiency of epididymal sperm, seminal anti-oxidase plasma activity, and the expression of copper-zinc superoxide dismutase (Cu-Zn SOD) (Zang et al., 2015). Additionally, the $\mathrm{Zn}$ nano-complex has improved the dose-dependent functionality of the spermatozoon plasma membranes without any detrimental effects on motility parameters. Studies have shown that in streptozotocin-induced diabetic rats, ZnO nanoparticles increase the activity of antioxidant enzymes in testicular tissue, increase sperm count and improve the properties of spermatozoa by protecting against oxidative stress (Falchia et al., 2018).

L-cysteine

L-cysteine (L-Cys) is a non-essential amino acid with a low molecular weight containing thiol. To engage in GSH biosynthesis both in vitro and in vivo, it achieves its effect on the cell membrane by easily penetrating the cell membrane. It protects membrane lipids and proteins by indirect scavenging of free radicals; it also acts as a membrane stabilizer and spermatozoon capacitation inhibitor (Amidi et al., 2016). L-cysteine has been shown to increase sperm motility, fertility, and morphology in bulls (Khan et al., 2021), ram (Andreea and Stela, 2010) and goats (Bucak and Uysal, 2010), and to enhance the chromatin structure and membrane integrity of pig semen stored by cooling. Çoyan et al. (2012) reported that cysteine improves Merino ram semen's mitochondrial activity after freeze-thaw without enhancing sperm motility. This can be metabolized to taurine after L-Cysteine passes through the cells. After a combination with a fatty acid in the plasma membrane, taurine transforms into acyl-taurine which improves surfactant properties and spermatozoon membrane osmoregulation (Amidi et al., 2016).

Selenium (Se)

Selenium (Se) is an important trace element well known to cells in both humans and animals. It acts as a key enzyme in the biological system in protection against oxidative stress by detoxifying free radicals by activating glutathione peroxidase and serves as a cofactor of glutathione synthetase. Selenium detoxifies the environment in the form of selenite in cell culture, to protect cells against oxidative damage (Amidi et al., 2016). Selenium supplementation in the diet has been reported to increase reproductive capacity in mice, sheep, and cattle (Amidi et al., 2016) and also to improve semen parameters after thawing Barbari goat frozen semen (Kumar et al., 2011). Reproductive problems and decreased semen quality resulting from selenium deficiency have been demonstrated in rats, rodents, pigs, rams, and cattle (Amidi et al., 2016).

The addition of selenium before freezing substantially increased the motility of bull semen (Amidi et al., 2016). Dorostkar et al. (2012) reported that diluents containing 1 and $2 \mu \mathrm{g} \mathrm{m}^{-1}$ Se significantly improved sperm motility, viability, membrane integrity, and total antioxidant capacity in sperm, and less damage was caused to the DNA of spermatozoa. It was also observed that selenium effects occurred in a dosedependent manner and harmful effects occurred on semen parameters at 4 to $8 \mu \mathrm{g} \mathrm{mL}^{-1}$ levels.

Many studies have used nano-selenium (SeNPs) as ROS scavengers to protect against oxidative damage in spermatozoa. The addition of Nano-Se to the semen extender improved the post-thaw efficiency and 
oxidative semen variables in a study on male semen. Furthermore, the oral treatment of Se-nanoparticles preserves the quality of spermatozoa (motility, DNA integrity) and spermatogenesis from oxidative damage caused by Cisplatin, a toxic anticancer agent on male reproduction (Safa et al., 2016).

Melatonin (MLT)

Melatonin is an indoleamine with two side chains, 5-methoxy group and 3-amide group. This can act as an antioxidant with its molecular weight of $232.2 \mathrm{~g} / \mathrm{mol}$, as well as regulate the biological clock and seasonal reproduction. MLT can protect different biomolecules from ROS damage by acting as a direct scavenger to detoxify reactive oxygen and nitrogen species through an easy crossing of cell membranes and the blood-brain barrier (Bhattacharya et al., 2019). It may also have indirect effects by stimulating various antioxidant enzymes such as melatonin, glutathione peroxidase/reductase, catalase, and superoxide dismutase. MLT demonstrates its powerful nonenzymatic antioxidant properties by removing various reactive oxygen and nitrogen species in vivo and in vitro. MLT has enhanced the characteristics of goats (Samir et al., 2020), rats (Sönmez et al., 2007), boar (Rocco et al., 2018), rams (Sarabi et al., 2009) and human sperm (Ortiz et al., 2010), according to results from some studies. The melatonin concentration needed to exert antioxidant effects on stored semen depends on the species of the animal, and this concentration varies from $0.01 \mathrm{mM}$ to $3.00 \mathrm{mM}$ (Medrano et al., 2017).

\section{Curcumin}

Curcumin is formulated as [1,7-bis (4-hydroxy-3methoxyphenyl) -1,6-heptadiene-3,5-dione] (CUR). Curcumin is the phytochemical that gives a yellow color to the turmeric and is now considered therapeutic. Curcumin demonstrates its antioxidant properties by scavenging different types of reactive oxygen, including radicals of superoxide anions, hydroxyl radicals, and radicals of nitrogen dioxides. Lipid peroxidation has also been shown to be inhibited in different animal models. At the same time, there are conflicting reports concerning the effects of curcumin on the parameters of male fertility. Many in vivo and in vitro studies illustrate its role in the energy-promoting and protective effects of curcumin on testicular tissue, spermatogenesis, and spermatozoa oxidative balance (Tvrda et al., 2015).

\section{Conclusion}

Cryopreservation of sperm is an applicable technique among assisted reproduction methods. However; some post-thaw semen parameters, including morphology, motility, viability, and DNA integrity, can be affected by cryopreservation. It is well known that antioxidants can reduce the negative effects of oxidative stress on the processing and development of the spermatozoa and embryos. The use of antioxidants has gained popularity for this reason because it prevents the formation of oxidation during semen freezing, minimizes the harmful impact of ROS and increases semen quality after thawing. Oxidative stress evaluation and the use of antioxidants are not regularly conducted in clinical practice. The explanation for this situation could be that antioxidant protective effects could not be completely identified, and their effect on pregnancy levels in the field could not be confirmed. It should also be recalled that antioxidants are costly chemicals. Therefore, further studies are required to determine an optimal freezing procedure for a species' semen and the appropriate antioxidant to be used.

\section{References}

Agarwal, A., \& Majzoub, A. (2017). Role of antioxidants in Assisted Reproductive Techniques. World J. Mens. Health. 35: 77-93. https://doi.org/10.5534/wjmh. 2017.35.2.77

Agarwal, A., Durairajanayagam, D., \& Du Plessis, S. S. (2014). Utility of antioxidants during assisted reproductive techniques: an evidence based review. Reproductive Biology and Endocrinology, 12(1), 1-19.

Agarwal, A., Makker, K., \& Sharma, R. (2008). "Clinical relevance of oxidative stress in male factor infertility: an update," American Journal of Reproductive Immunology. 59: 2-11. https://doi.org/10.1111/j.16000897.2007.00559.x

Aitken, R. J. (2017). Reactive oxygen species as mediators of sperm capacitation and pathological damage. Molecular reproduction and development, 84(10), 1039-1052. https://doi.org/10.1002/mrd.22871

Amidi, F., Pazhohan, A., Nashtaei, M.S., Khodarahmian, M., \& Nekoonam, S. (2016). The role of antioxidants in sperm freezing: a review. Cell Tissue Bank. 31: 330-9. https://doi.org/10.1007/s10561-016-9566-5.

Andreea, A., \& Stela, Z. (2010). Role of antioxidant additives in the protection of the cryopreserved semen against free radicals. Rom Biotechnol Lett. 15: 33-41.

Atmaca, G. (2004). Antioxidant effects of sulfur-containing amino acids. Yonsei Medical Journal. 45: 776-88. https://doi.org/10.3349/ymj.2004.45.5.776

Ball, B.A. (2008). Oxidative stress, osmotic stress and apoptosis: impacts on sperm function and preservation in the horse. Anim. Reprod. Sci. 107: 257-67. https://doi.org/10.1016/j.anireprosci.2008.04.014

Bansal, A.K., \& Bilaspuri, G.S. (2010a) Impacts of Oxidative Stress and Antioxidants on Semen Functions. SAGEHindawi Access to Research Veterinary Medicine International. Volume 2011. https://doi.org/10.4061/ 2011/686137

Bansal, A.K., \& Bilaspuri, G.S. (2010b). Effect of vitamin E and $\mathrm{Mn2}+$ on the thiol status of cattle bull spermatozoa 
under induced oxidative stress. Animal Science Papers and Reports. 28: 335-45.

Bello, T.K., Oyelowo, B.B., Khumran, A.M., Hassan, R., OkeEbgodo, B.E., Idris, S.Y., Aliyu, M.A., \& Maikaji, F. (2020). Antioxidative roles of Glutathione, Butylated Hydroxytoluene and Melatonin in semen preservation- $A$ Review. Nig. J. Anim. Sci. Tech. 3(1): 130-9.

Bhattacharya, K., Sengupta, P., \& Dutta, S. (2019). Role of melatonin in male reproduction. Asian Pacific Journal of Reproduction, 8(5), 211.

Birben, E., Sahiner, U.M., Sackesen, C., Erzurum, S., \& Kalayci, O. (2012). Oxidative stress and antioxidant defense. World Allergy Organ J. 5: 9-19. https://doi.org/ 10.1097/WOX.0b013e3182439613

Bollwein, H., \& Bittner, L. (2018). Impacts of oxidative stress on bovine sperm function and subsequent in vitro embryo development. Anim. Reprod. 15: 703-10. http://dx.doi.org/10.21451/1984-3143-AR2018-0041

Bollwein, H., Fuchs, I., \& Koess, C. (2008). Interrelationship between plasma membrane integrity, mitochondrial membrane potential and DNA fragmentation in cryopreserved bovine spermatozoa. Reprod Domest Anim. 43:189-95. https://doi.org/10.1111/j.14390531.2007.00876.x.

Bucak, M.N., Sarıozkan, S., Tuncer, P.B., Ateşşahin, A., Kulaksız, R., \& Çevik, M. (2010). The effect of antioxidants on postthawed Angora goat (Capra hircus ancryrensis) spermparameters, lipid peroxidation and antioxidant activities. Small Ruminant Research. 89, 24-30. https://doi.org/10.1016/j.smallrumres.2009.11.015

Cadet, J., Douki, T., Gasparutto, D., \& Ravanat, J. (2003). Oxidative damage to DNA: formation, measurement and biochemical features. Mutat Res Mol Mech Mutagen. 531:

5-23.

https://doi.org/10.1016/j.mrfmmm.2003.09.001

Çoyan, K., Bucak, M.N., Başpınar, N., Taşpınar, M., \& Aydos, S. (2012). Ergothioneine attenuates the DNA damage of post-thawed Merino ram sperm. Small Rumin. Res. 106: 165-7.

https://doi.org/10.1016/j.smallrumres.2012.02.002

Desai, N.R., Mahfouz, R., Sharma, R., Gupta, S., \& Agarwal, A. (2010). Reactive oxygen species levels are independent of sperm concentration, motility, and abstinence in a normal, healthy, proven fertileman: a longitudinal study. FERTIL STERIL. 94: 1541-43. https://doi.org/10.1016/ j.fertnstert.2009.12.041

Dorostkar, K., Alavi Shoushtari, S.M., \& Khaki, A. (2014). Effects of in vitro zinc sulphate additive to the semen extender on water buffalo (Bubalus bubalis) spermatozoa before and after freezing. International Journal of Fertility \& Sterility. 8(3): 325-32.

Dorostkar, K., Alavi-Shoushtari, S.M., \& Mokarizadeh, A. (2012). Effects of in vitro selenium addition to the semen extender on the spermatozoa characteristics before and after freezing in water buffaloes (Bubalus bubalis). Veterinary Research Forum, Faculty of Veterinary Medicine, Urmia University, Urmia, Iran. 4: 263.

Drevet, J.R., \& Aitken, R.J. (2020). Oxidation of Sperm Nucleus in Mammals: A Physiological Necessity to Some Extent with Adverse Impacts on Oocyte and Offspring.
Antioxidants. 9: 95. https://doi.org/10.3390/antiox 9020095

Falchia, L., Khalil, W.A., Hassan, M., \& Marei, W.F.A. (2018). Perspectives of nanotechnology in male fertility and sperm function. International Journal of Veterinary Science and Medicine. 6: 265-69. https://doi.org/ 10.1016/j.ijvsm.2018.09.001

Farshad, A., Amidi, F., Khor, A.K., \& Rashidi, A. (2011). Effect of Cholesterol-loaded cyclodextrin in presence and absence of egg yolk during freezing step on quality of Markhoz Buck's Spermatozoa. Asian- Australasian Journal of Animal Science. 4: 181-9. https://doi.org/10.5713/ajas.2011.10141

Félix, F., Oliveira, C. C., \& Cabrita, E. (2021). Antioxidants in Fish Sperm and the Potential Role of Melatonin. Antioxidants, 10(1), 36. https://doi.org/10.3390/antiox10010036

Gadea, J., Garcıa-Vazquez, F., Matas, C., Gardon, J.C., Canovas, S., \& Gumbao, D. (2005) Cooling and freezing of boar spermatozoa: supplementation of the freezing media with reduced glutathione preserves sperm function. Journal of Andrology. 26: 396-404. https://doi.org/ 10.2164/jandrol.04155

Gonçalves, F., Barretto, L.S.S., Arruda, R.P., Perri, S.H.V., \& Mingoti, G.Z. (2010). Effect of antioxidants during bovine in vitro fertilization procedures on spermatozoa and embryo development. Reproduction in Domestic Animals. 45: 129-35. https://doi.org/10.1111/j.14390531.2008.01272.x

Hezavehei, M., Sharafi, M., Kouchesfahani, H. M., Henkel, R., Agarwal, A., Esmaeili, V., \& Shahverdi, A. (2018). Sperm cryopreservation: A review on current molecular cryobiology and advanced approaches. Reproductive Biomedicine Online, 37(3), 327-339. https://doi.org/ 10.1016/j.rbmo.2018.05.012

Hussain, M., Begum, S.S., Kalita, M.K., Ahmed, K.U., \& Nath, R. (2018). Additives used in semen preservation in animals: A short review. International Journal of Chemical Studies. 6(5): 354-361.

Ifeanyi, O. E. (2018). A review on free radicals and antioxidants. Int. J. Curr. Res. Med. Sci, 4(2), 123-133.

Khan, I. M., Xu, D., Cao, Z., Liu, H., Khan, A., Rahman, S. U., \& Zhang, Y. (2021). Addition of L-Cysteine and Vitamin E to Semen Diluent Enhances Freeze-thawed Spermatozoa Characteristics in Crossbred Cattle Bulls under Subtropical Environment. Pakistan Journal of Zoology. 111.

Kumar, T., Kumar, A., Swain, D.K., \& Yadav, S. (2011). Influence of oral supplementation of Zinc and Selenium on post thaw semen quality of Barbari bucks. Journal of Animal Research. 1: 41-6.

Lambert, I.H., Pedersen, S.F., \& Poulsen, K.A. (2006). Activation of PLA2 isoforms by cell swelling and ischaemia/hypoxia. Acta Physiol. 187: 75-85. https://doi.org/10.1111/ j.1748-1716.2006.01557.x

Martinez-Paramo, S., Diogo, P., Dinis, M., Herraez, M., Sarasquete, C., \& Cabrita, E. (2012). Incorporation of ascorbic acid and atocopherol to the extender media to enhance antioxidant system of cryopreserved sea bass sperm. Theriogenology. 77: 1129-36. https://doi.org/ 10.1016/j.theriogenology.2011.10.017 
McCarthy, M.J., Baumber, J., Kass, P.H., \& Meyers, S.A. (2010). Osmotic stress induces oxidative cell damage to rhesus macaque spermatozoa. Biol. Reprod. 82:644-51. https://doi.org/10.1095/biolreprod.109.080507

Medrano, A., Contreras, C.F., Herrera, F.M., \& AlcantarRodriguez, A.M. (2017). Melatonin as an antioxidant preserving sperm from domestic animals. Asian Pacific Journal of Reproduction. 6: 241-6. https://doi.org/ 10.4103/2305-0500.217317

Moazamian, R., Polhemus, A., Connaughton, H., Fraser, B., Whiting, S., Gharagozloo, P., \& Aitken, R.J. (2015). Oxidative stress and human spermatozoa: diagnostic and functional significance of aldehydes generated as a result of lipid peroxidation. Mol Hum Reprod. 21: 50215. https://doi.org/10.1093/molehr/gav014

Mora-Esteves, C., \& Shin, D. (2013). Nutrient supplementation: improving male fertility fourfold. Seminars in Reproductive Medicine. 31: 293-300. https://doi.org/ 10.1055/s-0033-1345277

Ortiz, A., Espino, J., Bejarano, I., Lozano, G.M., Monllor, F., García, J.F., Pariente, J.A., \& Rodríguez, A.B. (2010). High endogenous melatonin concentrations enhance sperm quality and short-term in vitro exposure to melatonin improves aspects of sperm motility. Journal of Pineal Research. 50: 132-139. https://doi.org/10.1111/j.1600079X.2010.00822.x

Rocco, M., Betarelli, R., Placci, A., Fernández-Novell, J. M., Spinaci, M., Casao, A., ... \& Rodríguez-Gil, J. E. (2018). Melatonin affects the motility and adhesiveness of in vitro capacitated boar spermatozoa via a mechanism that does not depend on intracellular ROS levels. Andrology, 6(5), 720-736.

Safa, S., Moghaddam, G., Jozani, R.J., Kia, H.D., \& Janmohammadi, H. (2016). Effect of vitamin E and selenium nanoparticles on post-thaw variables and oxidative status of rooster semen. Animal Reproduction Science. 174:100-6. https://doi.org/10.1016/ j.anireprosci. 2016.09.011

Samir, H., Nyametease, P., Elbadawy, M., Nagaoka, K., Sasaki, K., \& Watanabe, G. (2020). Administration of melatonin improves testicular blood flow, circulating hormones, and semen quality in Shiba goats. Theriogenology, 146, 111-119.

Sanocka, D., \& Kurpisz, M. (2004). Reactive oxygen species and sperm cells. Reproductive Biology and Endocrinology.2:12-26. https://doi.org/10.5772/ intechopen.73037

Silva, S., Soares, A., Batista, A., Almeida, F., Nunes, J., Peixoto, C., \& Guerra, M. (2011). In vitro and in vivo evaluation of ram sperm frozen in tris egg-yolk and supplemented with superoxide dismutase and reduced glutathione. Reprod. Domest. Anim. 46: 874-81. https://doi.org/10.1111/ j.1439-0531.2011.01758.x.

Sönmez, M., Yüce, A., \& Türk, G. (2007). The protective effects of melatonin and vitamin $E$ on antioxidant enzyme activities and epididymal sperm characteristics of homocysteine treated male rats. Reprod. Toxicol. 23: 226-31. https://doi.org/10.1016/j.reprotox.2006.11.003.

Stradaioli, G., Noro, T., Sylla, L., \& Monaci, M. (2007). Decrease in glutathione (GSH) content in bovine sperm after cryopreservation: comparison between two extenders. Theriogenology. 67:1249-55. https://doi.org/10.1016/ j.theriogenology.2007.01.009

Tvrda, E., Lukáč, N., Jambor, T., Lukáčová, J., \& Massányi, P. (2015). Curcumın In Male Fertility: Effects On Spermatozoa Vitality And Oxidative Balance. J Microbiol Biotech Food Sci / Tvrdá et al. 4: 120-124. https://doi.org/10.15414/jmbfs.2015.4.special2.120124

Uysal, O., Kinet, H., Çevik, M. ve Çetinkaya, S. (2000). Fertility obtained from frozen ram semen with different extenders containing varied antioxidants. Journal of Ankara University Faculty of Veterinary Medicine. 47 (2), 177-189.

Wathes, D.C., Abayasekara, D.R., \& Aitken, R.J. (2007). Polyunsaturated fatty acids in male and female reproduction. Biology of Reproduction. 77: 190-201. https://doi.org/10.1095/biolreprod.107.060558 